\title{
Formation of Neutron-Rich and Superheavy Elements in Astrophysical Objects
}

\author{
Rabinarayan Panda ${ }^{1}$, Suresh Kumar Patra ${ }^{2}$ \\ ${ }^{1}$ Department of Physics, ITER, Siksha O Anusandhan University, Bhubaneswar, India \\ ${ }^{2}$ Institute of Physics, Sachivalaya Marg, Bhubaneswar, India \\ E-mail:rnpanda@iopb.res.in,patra@iopb.res.in \\ Received August 9, 2010; revised September 13, 2010; accepted September 9, 2010
}

\begin{abstract}
We calculate the reaction and the fusion cross-sections of neutron-rich heavy nuclei taking light exotic isotopes as projectiles. Results of neutron-rich $\mathrm{Pb}$ and $\mathrm{U}$ isotopes are demonstrated as the representative targets and $\mathrm{He}, \mathrm{B}$ as the projectiles. The Gluaber Model and the Coupled Channel Formalism are used to evaluate the reaction and the fusion cross-sections for the cases considered. Based on the analysis of these cross-sections, we predict the formation of heavy, superheavy and super-superheavy elements through rapid neutron/ light nuclei capture r-process of the nucleosynthesis in astrophysical objects.
\end{abstract}

Keywords: Total Nuclear Cross Section, Fusion Reaction Cross Section, Gluaber Model, Coupled Channel Formalism

\section{Introduction}

Formation of superheavy elements (SHE) in the laboratory is one of the most challenging problems in Nuclear Physics. So far the synthesis of $Z=118$ element has been possible [1]. Efforts are on to synthesize still heavier elements in various laboratories all over the world. It is certain that if an element is created through human efforts then most probably it may be present naturally somewhere in the Universe. Thus the mode of formation of superheavy or super-superheavy element in astrophysical object is a fundamental question in the field of Nuclear Astrophysics. In this context, it is likely that the superheavy element with $\mathrm{Z}$ $=118$ and higher atomic numbers are present. It has been reported in Ref. [2], and the stability of the most stable superheavy elements could be as high as $10^{9}$ years in some of the calculations [3-7]. Thus, the study of unstable nuclei with radioactive ion beam (RIB) facilities has opened an exciting channel to look up to some of the crucial issues in the context of both nuclear structure and astrophysics [8]. Unstable nuclei play an influential, and in some cases dominant role.

The direct study of stellar properties in ground-based laboratories has become feasible, due to the availability of RIBs; for example the study of ${ }^{18} \mathrm{Ne}$ induced neutron pick-up reaction could reveal inform-tion about the exotic ${ }^{15} \mathrm{O}+{ }^{19} \mathrm{Ne}$ reaction occurring in the $\mathrm{CNO}$ cycle in stars. Study of the structure and the reactions of not only unstable light exotic but also of the superheavy and the super-superheavy nuclei is therefore required to improve our understanding of the astrophysical origin of atomic nuclei and the evolution of stars and their death, because, the formation of neutron-rich/super-superheavy nuclei determine the endpoint of the rapid-neutron (rn-) capture process in nucleosynthesis.

In a recent study, Satpathy et al. [9] claimed the neutron-rich $\mathrm{U}$ and Th-isotopes are thermally fissile and could release orders of magnitude more energy than ${ }^{235} \mathrm{U}$ in a new mode of fission decay called multi-fragmentation fission, which happened frequently in astrophysical objects, which may cause the termination of the rn-capture process. The main objective of the present letter is to study the reaction $\left(\sigma_{r}\right)$ and fusion $\left(\sigma_{f}\right)$ cross-sections of neutron-rich $U$ and some other interesting exotic isotopes, which are related to the formation of neutron-rich, SHE and super-SHE elements in the Universe. The value of $\sigma_{r}$ is calculated by using the most recently developed effective field theory motivated relativistic mean field (ERMF) nuclear densities [10-13], in conjunction with the Glauber model. However, $\sigma_{f}$ is estimated in the nonrelativistic coupled channel calculation. From the calculated reaction and fusion cross-sections, we look for the formation path of neutron rich, SHE and super-SHE nuclei in the cosmos. 


\section{Theoretical Formalism}

The theoretical formalism to calculate the nuclear reaction cross-section using Glauber approach has been given by R. J. Glauber [14]. The standard Glauber form for the reaction cross-section at high energies is expressed [14] as:

$$
\sigma_{r}=2 \pi \int_{0}^{\infty} b[1-T(b)] d b,
$$

where $T(b)$, the transparency function, is the probability that at an impact parameter $b$ the projectile passes through the target without interaction. This function $T(b)$ is calculated in the overlap region between the projectile and target where the interactions are assumed to result from single nucleon-nucleon collision and is given by

$$
T(b)=\exp \left[-\sum_{i, j} \bar{\sigma}_{i j} \int d \vec{s} \bar{\rho}_{t i}(s) \bar{\rho}_{p j}(|\vec{b}-\vec{s}| s)\right]
$$

Here, the summation indices $i, j$ run over proton and neutron numbers and subscript $p$ and $t$ refer to projectile and target respectively.

The original Glauber model is designed for high energy projectile, like relativistic proton reactions. It fails to describe the collisions induced at relatively low energies. In this case, the straight-line trajectory is modified because of the presence of the Coulomb field of the target and projectile. In such cases the present version of Glauber model is modified in order to take care of finite range effects [15] in the profile function and the Coulomb modified trajectories. Thus for finite range approximations, the transparency function is given by

$$
\begin{aligned}
T(b) & =\exp \left[-\int_{P T} \sum_{i, j}\left[\Gamma_{i j}(\vec{b}-\vec{s}+\vec{t})\right]\right. \\
& \left.\times \bar{\rho}_{P i}(\vec{t}) \bar{\rho}_{T j}(\vec{s}) d \vec{s} d \vec{t}\right],
\end{aligned}
$$

Here the profile function $\Gamma_{i j}$ is given by

$$
\Gamma_{i j}\left(b_{e f f}\right)=\frac{1-i \alpha}{2 \pi \beta_{N N}^{2}} \sigma_{i j} \exp \left(-\frac{b_{e f f}^{2}}{2 \beta_{N N}^{2}}\right),
$$

where $b_{\text {eff }}=|\vec{b}-\vec{s}+\vec{t}|, \vec{b}$ is the impact parameter, $\vec{s}$ and $\vec{t}$ are just the dummy variables for integration over the z-integrated target and projectile densities. The values of the parameters, $\bar{\sigma}_{i j}, \alpha$ and $\beta_{N N}$ are taken from Ref. [16-18]. The detailed formalism is available in Ref. [19-21]. The E-RMF density with G2 parameter set $[10-13,22,23]$ is used as input for the evaluation of $\sigma_{r}$. For the details of the calculation of ground state properties of finite nuclei and the procedure of estimation of nuclear reaction cross-section, we refer the reader to Refs. [19-24]. To compute the fusion cross-section $\sigma_{f}$ we fol- low the coupled-channel calculations including all orders of coupling. This is done in a non-relativistic framework. The computer code CCFULL as developed in Ref. [25] is used. The fusion cross-section is given by the formula [25]:

$$
\sigma_{f}(E)=\sum_{J} \sigma_{J}(E)=\frac{\pi}{k_{0}^{2}} \sum_{J}(2 J+1) P_{J}(E),
$$

with $P_{J}(E)$ is the inclusive penetrability and the other symbols have the standard meaning as defined in [25].

\section{Calculations and Results}

It was shown in our earlier papers that the densities taken from relativistic mean field formalism, and used in the frame-work of Glauber model $[14,24]$ to evaluate the differential and total reaction cross-section is quite successful for light systems [19-21]. Now we extend the model to calculate the total reaction cross-section considering light exotic nuclei as projectile and heavy neutron-rich isotopes as target. Here, we calculate as the representative cases for the reaction cross-section of neutron-rich $\mathrm{Pb}$ and $\mathrm{U}$ isotopes taking exotic $\mathrm{He}$ and $\mathrm{B}$ nuclei as incident projectile. In Figure $\mathbf{1}$ the reaction cross-section $\sigma_{r}$ for ${ }^{4} \mathrm{He}+{ }^{208,228,248,278} \mathrm{~Pb},{ }^{10,15,17,20} \mathrm{~B}+$ ${ }^{208} \mathrm{~Pb},{ }^{4} \mathrm{He}+{ }^{235,250,270,290} \mathrm{U}$ and ${ }^{10,15,17,20} \mathrm{~B}+{ }^{235} \mathrm{U}$ are presented. From the calculated results, the increase in $\sigma_{r}$ is quite substantial with the target mass. The same observation is also applicable, while increasing the mass of the projectile (keeping the target mass constant). In any of these cases, the reaction cross-section becomes favorable with either increase of projectile mass or the mass of the target or both. The enhancement can be understood by the simple geometrical area of the nucleus $\pi R^{2}$, where $R$ is the sum of the radii of the target and the projectile. The nuclear radius with the mass number is connected with the relation $\mathrm{R}=r_{0} A^{1 / 3}$, where $r_{0}=1.36 \mathrm{fm}$ and one expect $\sigma_{r} \propto\left(A_{t}^{1 / 3}+A_{p}^{1 / 3}\right)^{2}$. Bradt and Peters [26] modified this relation to take into account the deviation from the experimental systematic and it is expressed as

$\sigma_{r}=\pi r_{0}^{2}\left[\left(A_{t}^{1 / 3}+A_{p}^{1 / 3}\right)-b_{0}\right]^{2}$, where $b_{0}=2.247-0.915$ $\left(A_{p}^{-1 / 3}-A_{t}^{-1 / 3}\right)$. This formula is further improved in $[27,28]$ and later on the Coulomb correction was included $[29,30]$. The semi-empirical formula to calculate the total nuclear reaction cross-section [31,32] and experimental measurements $[33,34]$ also shows the size dependence of $\sigma_{r}$ on the masses of target and projectile $[33,34]$. This implies the probability of formation of heavier masses in the reaction process with heavier isotope of the projectile as well as target. In Ref. [35], within the formalism of a Thomas-Fermi model, calculations are presented for nu- 
clei beyond the nuclear drip-line at zero temperature. This is possible because of the presence of an external neutron gas which may be envisaged in the astrophysical scenario and is the situation of the present discussion for accreting cosmological objects.

In Figure 2 the fusion cross section $\sigma_{f}$ for various neutron-rich light nuclei with heavier drip-line isotopes like ${ }^{4} \mathrm{He}+{ }^{208,228,248,278} \mathrm{~Pb},{ }^{10,15,17,20} \mathrm{~B}+{ }^{208} \mathrm{~Pb},{ }^{4} \mathrm{He}+{ }^{235,250,270,290} \mathrm{U}$

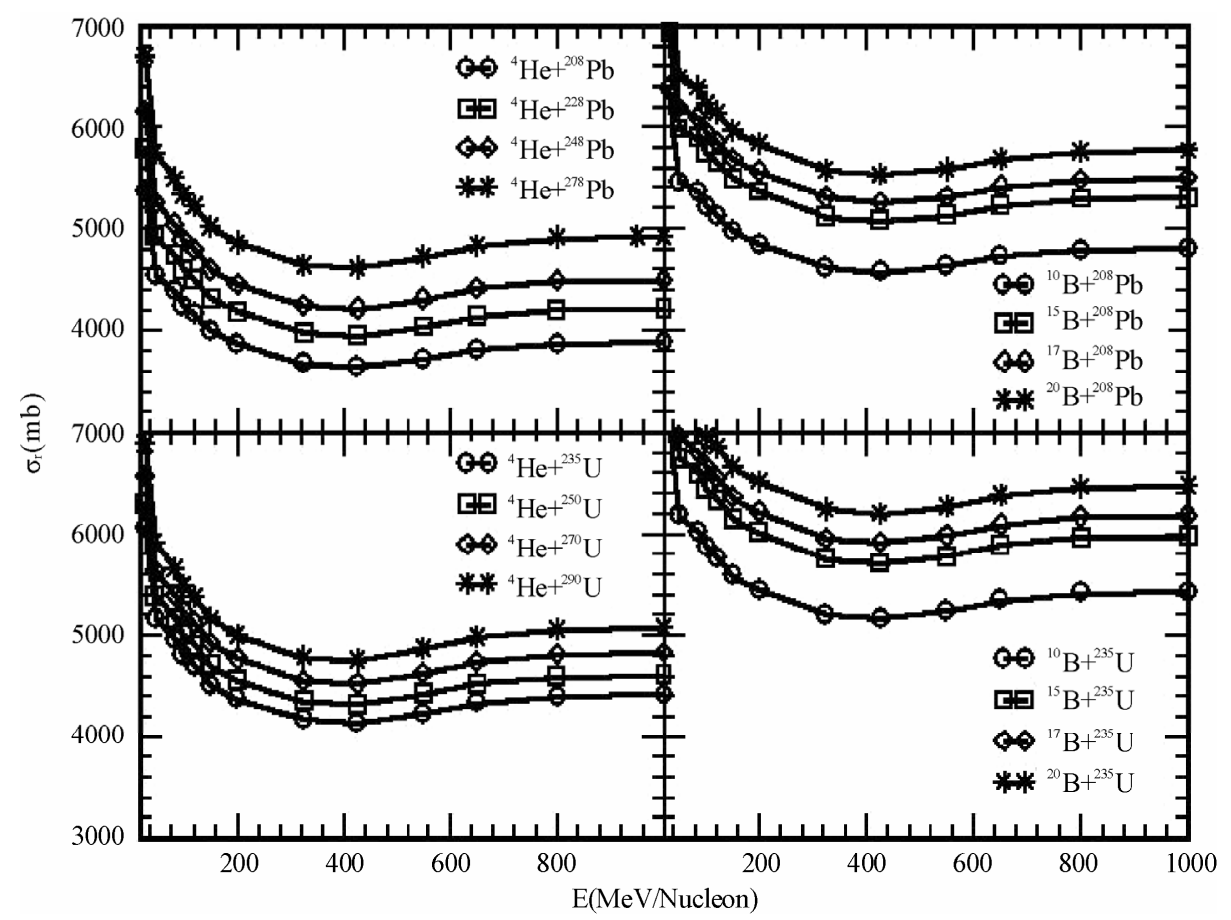

Figure 1. The nuclear reaction cross-sections taking $\mathrm{He}$ and $\mathrm{B}$ isotopes as projectile with different isotopes of $\mathrm{Pb}$ and $\mathrm{U}$.

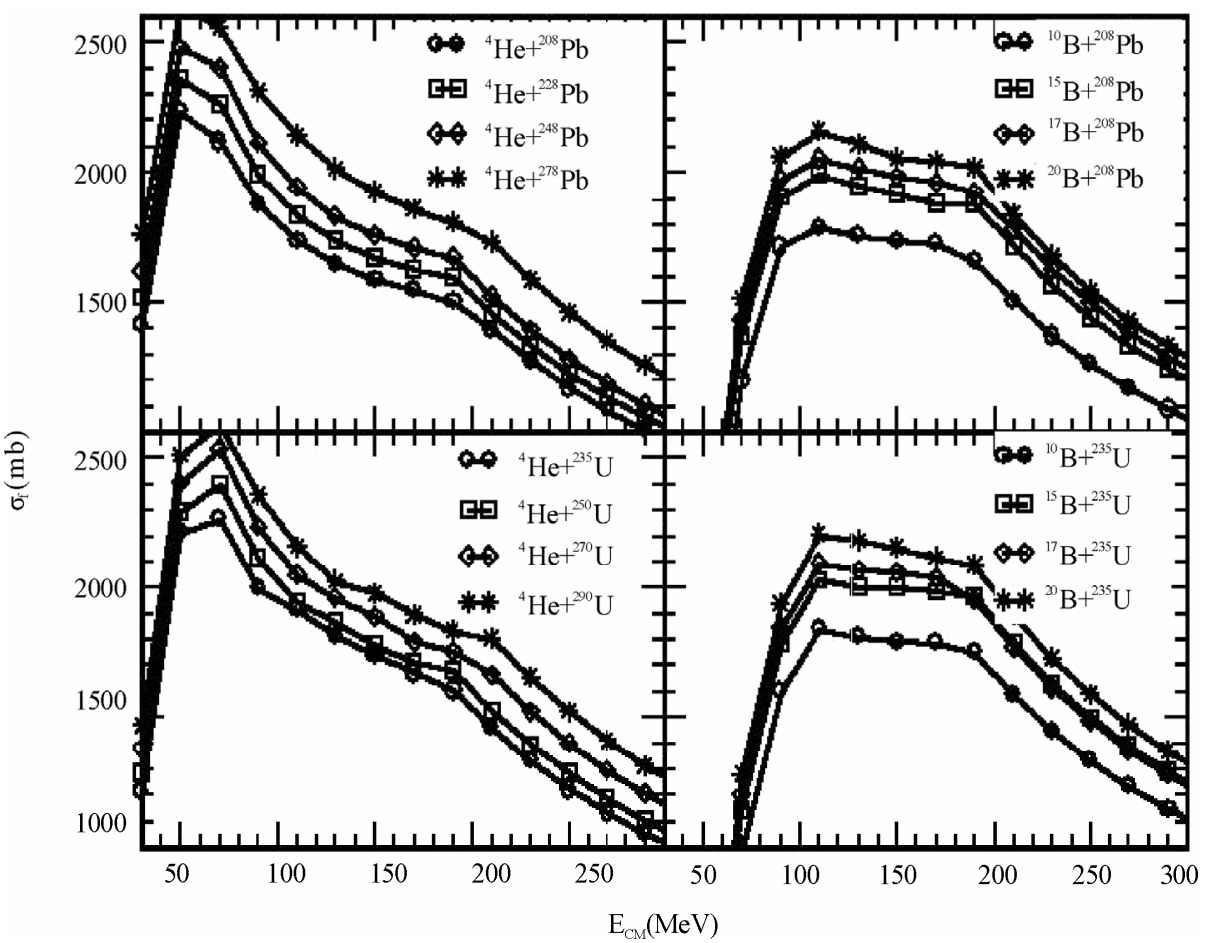

Figure 2. The nuclear fusion cross-sections taking $\mathrm{He}$ and $\mathrm{B}$ isotopes as projectile with different isotopes of $\mathrm{Pb}$ and $\mathrm{U}$. 
and ${ }^{10,15,17,20} \mathrm{~B}+{ }^{235} \mathrm{U}$ are shown. Similar to the reaction cross-section, the increase in $\sigma_{f}$ is quite clear with the increase of target, projectile or both the masses. This implies the probability of creation of heavier masses with the increase of mass number of the projectile as well as target and making the way for the evolution of neutronrich heavy nuclei much beyond the drip-line [35] due to the presence of the external neutron gas or highly neutron-rich light as well as heavy nuclei generates in the astrophysical objects.

Analysis of Figures 1 and 2 shows that, the magnitude of $\sigma_{r}$ and $\sigma_{f}$ is optimum at $\sim 30$ to $200 \mathrm{MeV}$ of the incident projectile energy. Beyond this range, the value of $\sigma_{r}$ and $\sigma_{f}$ decreases drastically. Both the cross-sections indicate the suitability of the incident projectile energy for a favorable condition of the formation of the fused elements in the astrophysical system. Thus, the chance of the formation of heavier element is maximum, if a suitable energy range is created. A possible system for such case may be relativistic jets of Gamma Ray Burst (GRBs) or supernovae jets near the nascent neutron star [36,37]. The high energy environment in such cosmological objects is because of the supernova shock [38] and it is quite common in the nascent neutron star or relativistic jets of GRBs [36,37].

In these objects a highly neutron-rich and high temperature scenario is made possible and which may be a probable platform for such reactions. In this context, it is worth citing the following example: A neutron star is burned when a star of mass $\sim 20 \mathrm{M}_{\odot}$ undergoes its core collapses after hyper-energetic explosions of Gamma ray bursts. A star with initially $\sim 20 \mathrm{M}_{\odot}$ would develop carbon-oxygen core of $\sim 3.3 \mathrm{M} \odot$. It left behind a neutron star of $\sim 1.4 \mathrm{M} \odot, \sim 1.3 \mathrm{M} \odot$ of oxygen and $\sim 0.6 \mathrm{M} \odot$ of heavier elements, $\mathrm{Si}$ and Fe group, which could be ejected in the supernova. Such a collapse gives rise to an explosion of kinetic energy K. E. $\sim 10^{51} \operatorname{ergs}\left(\sim 6.25 \times 10^{56} \mathrm{MeV}\right)$ $[36,37]$. Young neutron stars have a fluid surface, a solid, crystalline crust and a fluid interior. The fluid regions of the star adjust themselves to its rotation which remaining always asymmetric. The radiated power comes directly from the rotational energy of the neutron star. The entropy in mass elements exhibiting the neutron star at later times will be larger than the earlier. This is because, most of the heating occurs near the surface of the neutron star. Slowly with time the radius of the neutron star shrinks from 100 $\mathrm{Km}$ to $10 \mathrm{Km}[39,40]$. The decrease in the initial radius start from which the mass elements begin increasing the heat rate $[36,37]$. It is worth mentioning of the burning process of $\mathrm{H}, \mathrm{He}, \mathrm{Li} \ldots$ in the accreting astrophysical system. To maintain hydrostatic equilibrium [41], this continues up to formation of Iron. When this stage is reached, depending on its mass, the astrophysical object undergoes various phenomena like supernovae explosion, X-rays burst, GRBs, formation of neutron star, black hole, red giant or white dwarf etc. In some cases, it becomes highly neutron-rich/light-nuclei ( $\mathrm{He}, \mathrm{Li}, \mathrm{Be} . .$.$) environ-$ ment which is favorable for the fusion of such low mass nuclei and could makes the way for the formation of heavier isotopes. This process supposes to continue up to certain A or Z number. Slowly, this fusion process becomes less favorable, as they can not overcome the Coulomb barrier. After this stage, the rn-capture process gains importance which prolongs for a longer time for the formation of ultra-heavy nuclei.

Thus, in the course of time, the neutron-rich light element fused with these heavy nuclei and more heavier isotopes with a little increase of proton number is generated in the process; for example, ${ }^{4} \mathrm{He}+{ }^{208} \mathrm{~Pb}$ gives ${ }^{212} \mathrm{Po}$. Again ${ }^{212}$ Po reacts with ${ }^{4} \mathrm{He}$ to form ${ }^{216} \mathrm{Rn}$. A schematic diagram for the process of SHE formation is shown in Figure 3.

From the figure, it can be understood how this phenomenon goes on to create much heavier isotopes. Similarly other processes also continue to go on as shown in Figures 1, 2 and 3, such as ${ }^{20} \mathrm{~B}+{ }^{235} \mathrm{U} \rightarrow{ }^{255} \mathrm{Bk},{ }^{20} \mathrm{~B}+{ }^{255 \mathrm{~B}} \mathrm{k}$ $\rightarrow{ }^{275}$ No..... and so on. A representative example is depicted in Figure 4. As mentioned earlier, after the supernovae explosion, in the rn-process, heavy normal/ exotic nuclei including the ultra-neutron rich light isotopes are formed. Exotic nuclei like ${ }^{6} \mathrm{He},{ }^{11} \mathrm{Li},{ }^{14} \mathrm{Be},{ }^{20} \mathrm{~B}$, normal actinides (e.g. ${ }^{208} \mathrm{~Pb},{ }^{235} \mathrm{U}$ etc.) and neutron-rich drip-line isotopes, similar to ${ }^{278} \mathrm{~Pb}$ etc. are generated. Thereafter, fusion process of the light isotopes with heavier nuclei becomes important. The increase of fusion cross-sections as shown in Figure 4 confirmed the possibility of the formation of ultra-heavy isotopes as well as super heavy elements both with lower and higher atomic masses. The demonstration of a path for the formation of ${ }^{408} \mathrm{X}_{132}(\mathrm{~A}=408, \mathrm{Z}=132, \mathrm{~N}=276)$ through complete fusion process is given below (whose cross-sections are shown in Figure 4):

$$
\begin{aligned}
& { }^{20} \mathrm{~B}+{ }^{208} \mathrm{~Pb} \rightarrow{ }^{228} \mathrm{Fr},{ }^{20} \mathrm{~B}+{ }^{228} \mathrm{Fr} \rightarrow{ }^{248} \mathrm{U},{ }^{20} \mathrm{~B}+{ }^{248} \mathrm{U} \rightarrow{ }^{268} \mathrm{Bk}, \\
& { }^{20} \mathrm{~B}+{ }^{268} \mathrm{Bk} \rightarrow{ }^{288} \mathrm{No},{ }^{20} \mathrm{~B}+{ }^{288} \mathrm{No} \rightarrow{ }^{308} \mathrm{Bh},{ }^{20} \mathrm{~B}+{ }^{308} \mathrm{Bh} \rightarrow \\
& { }^{328} \mathrm{X}_{112},{ }^{20} \mathrm{~B}+{ }^{328} \mathrm{X}_{112} \rightarrow{ }^{348} \mathrm{X}_{117},{ }^{20} \mathrm{~B}+{ }^{348} \mathrm{X}_{117} \rightarrow{ }^{368} \mathrm{X}_{122}, \\
& { }^{20} \mathrm{~B}+{ }^{368} \mathrm{X}_{122} \rightarrow{ }^{388} \mathrm{X}_{127},{ }^{20} \mathrm{~B}+{ }^{388} \mathrm{X}_{127} \rightarrow{ }^{408} \mathrm{X}_{132} \text { and so on. }
\end{aligned}
$$

Thus, each time the proton number $\mathrm{Z}$ increases by 5 units the mass number $A$ goes up by 20 units in the case of ${ }^{20} \mathrm{~B}$ capture. Slowly, it creates a highly neutron-rich heavy isotope, which is enabled to capture any more neutron $\mathrm{n}$ or neutron-rich nucleus. This is termed as waiting point.

Here, the neutron-rich heavy element emits a $\beta^{-}$particle, and the daughter nucleus gains a positive charge by converting a neutron (n) to a proton (p). Due to this en- 


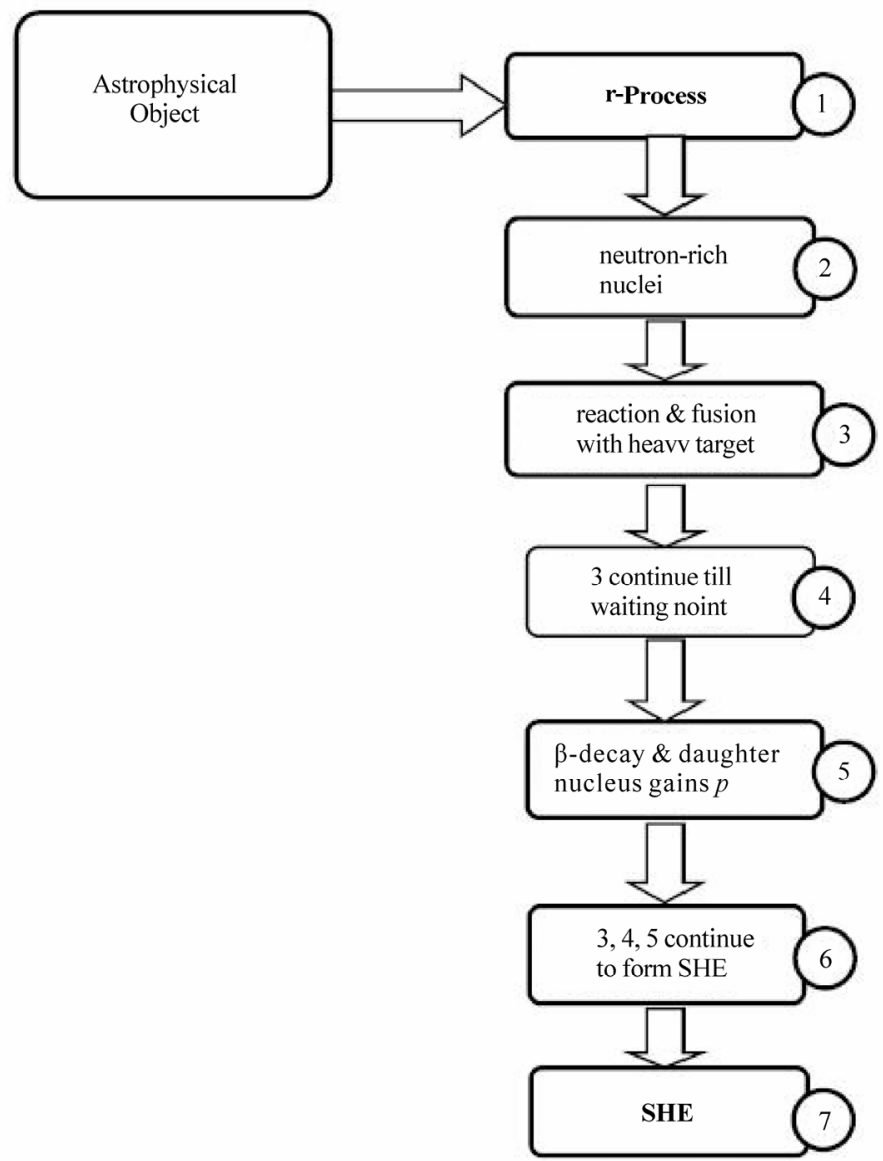

Figure 3. The schematic diagram for the formation of super heavy element (SHE) in the astrophysical object. The production of SHE is possible through reaction and fusion processes at a favorable energy condition in the cosmos.

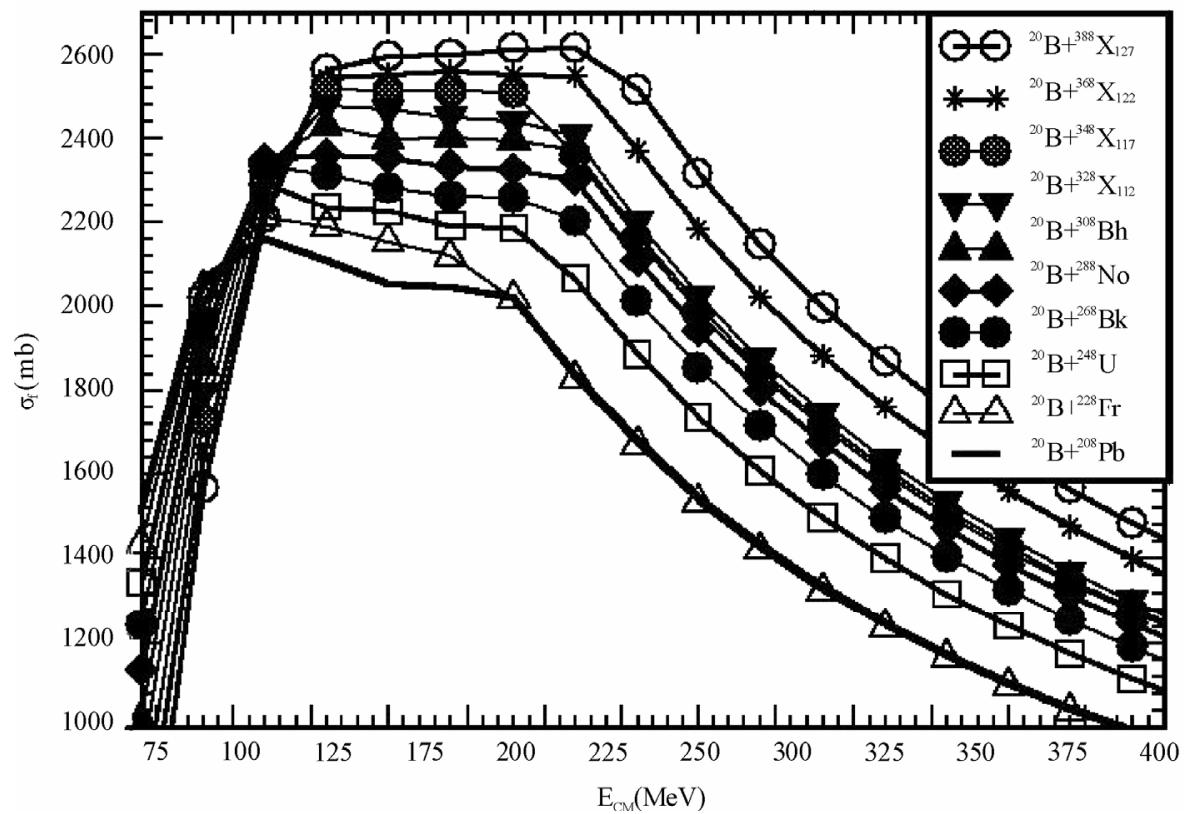

Figure 4. A representative paths for the formation of ${ }^{408} X_{132}$ superheavy elements through ${ }^{20} B$ capture process. The fusion cross-sections for $\sigma_{f}$ various daughter nuclei with ${ }^{20} \mathrm{~B}$ are shown. 
hancement in $\mathrm{Z}$, the product (daughter nucleus) captures few more $\mathrm{n}$ or neutron-rich light nuclei by fusion process till it reaches the new waiting point. At this point, the nucleus gains another proton $\mathrm{p}$, by emitting $\beta^{-}$particle. This process continues and SHE or super-SHE is formed in the cosmological object. In this context, it is worth mentioning that, the dominant modes of decays are $\beta^{-}$and spontaneous fission for large $\mathrm{N}$ and large $\mathrm{Z}$ nuclei, respectively. In the $\beta^{-}$decay, the daughter nucleus gains a proton, whereas for large $\mathrm{N}$, the spontaneous fission reduces considerably due to excess number of neutrons [9] and the neutron-rich isotope becomes fission stable as the height of the fission barrier decreases and the width increases, thereby making the nucleus more stable against fission [9]. It is interesting to mention here that, recently it has been reported by A. Marinov et al. $[42,43]$, that the evidence of a super heavy isotope with $Z=122$ or 124 and a mass number $A=292$; has been found in natural $\mathrm{Th}$ using inductively coupled plasma-sector field mass spectrometry. The estimated half-life of this isotope is $t_{1 / 2} \geq$ $10^{8}$ years, comparable with the theoretical predictions [3-7].

\section{Summary and Conclusions}

In summary, we estimated the reaction and fusion crosssection of various combinations of light and heavy isotopes. We extended the calculations to exotic systems taking into consideration the possibility of availing the rnprocess and the exotic nuclei capture processes in astrophysical objects. The enhanced cross-sections with increase of mass number for both the projectile and target made it possible for the formation of the heavier neutron-rich nuclei way beyond the normal drip lines predicted by the mass models. By the neutron or heavy ion (light neutron-rich nuclei) capture process the daughter nucleus becomes a super heavy element which may be available somewhere in the Universe in super-natural condition and can be possible to be synthesized in laboratories. Here the stability of the neutron-rich SHE or super-SHE against spontaneous fission arises due to widening of the fission barrier because of the excess number of neutrons.

\section{Acknowledgements}

Discussions with Professors K. Langanke, L. Satpathy and C. R. Praharaj are gratefully acknowledged. We are thankful to Prof. A. Abbas for a careful reading of the manuscript. This work has been supported in part by Council of Scientific \& Industrial Research (No. 03 (1060) 06/EMR-II) as well as the project No. SR/S2/ HEP-
16/2005, Department of Science and Technology, Govt. of India.

\section{References}

[1] Yu. Ts. Oganessian, et al., "Synthesis of the Isotopes of Elements 118 and 116 in the ${ }^{249} \mathrm{Cf}$ and ${ }^{245} \mathrm{Cm}+{ }^{48} \mathrm{Ca} \mathrm{Fu}-$ sion Reaction," Physical Review C, Vol. 74, No. 4, 2006, p. 044602 .

[2] P. Moller and J. R. Nix, "Stability and Decay of Nuclei at the End of the Periodic System," Nuclear Physics A, Vol. 549, No. 1, 1992, pp. 84-102.

[3] S. G. Nilsson, et al., "On the Spontaneous Fission of Nuclei with $Z$ near 114 and $N$ near 184," Nuclear Physics $A$, Vol. 115, No. 3, 1968, pp. 545-562.

[4] S. G. Nilsson, et al., "On the Nuclear Structure and Stability of Heavy and Superheavy Elements", Nuclear Physics A, Vol. 131, No. 1, 1969, pp. 1-66.

[5] M. Brack, J. Damgaaid, A. S. Jensen, H. C. Pauli, V. M. Strutinsky and C. Y. Wong, "Funny Hills: The ShellCorrection. Approach to Nuclear Shell Effects and Its Applications to the Fission Process," Reviews of Modern Physics, Vol. 44, No. 2, 1972, pp. 320-405.

[6] E. O. Fiset and J. R. Nix, "Calculation of Half-Lives for Superheavy Nuclei," Nuclear Physics A, Vol. 193, No. 2, 1972, pp. 647-671.

[7] J. R. Nix, "Calculation of Fission Barriers for Heavy and Superheavy Nuclei," Annual Review of Nuclear Science, Vol. 22, 1972, pp. 65-120.

[8] A. Ozawa, T. Suzuki and I. Tanihata, "Nuclear Size and Related Topics," Nuclear Physics A, Vol. 693, No. 1-2, 2001, pp. 32-62.

[9] L. Satpathy, S. K. Patra and R. K. Choudhury, "Fission Decay Properties of Ultra Neutron-Rich Uranium Isotopes," PRAMANA-Journal of Physics, Vol. 70, No. 1, 2008, pp. 87-99.

[10] R. J. Furnstahl, B. D. Serot and H. B. Tang, "Analysis of Chiral Mean-field Models for Nuclei," Nuclear Physics A, Vol. 598, No. 4, 1996, pp. 539-582.

[11] R. J. Furnstahl, B. D. Serot and H. B. Tang, "A Chiral Effective Lagrangian for Nuclei," Nuclear Physics A, Vol. 615, No. 3-4, 1997, pp. 441-482.

[12] B. D. Serot and J. D. Walecka, "Recent Progress in Quantum Hydrodynamics," International Journal of Modern Physics E, Vol. 6, No. 4, 1997, pp. 515-631.

[13] R. J. Furnstahl and B. D. Serot, "Parameter Counting in Relativistic Mean-field Models," Nuclear Physics A, Vol. 671, No. 1-4, 2000, pp. 447-460.

[14] R. J. Glauber, "Lectures on Theoretical Physics," In: W. E. Brittin and L. C. Dunham, Eds., Interscience, Vol. 1, New York, 1959, p. 315.

[15] P. Shula, "Glauber Model and the Heavy ion Reaction Cross Section," Physical Review C, Vol. 67, No. 5, 2003, p. 054607. 
[16] M. Y. H. Farag, "Modified Glauber Model for the Total Reaction Cross-section of ${ }^{12} \mathrm{C}+{ }^{12} \mathrm{C}$ Collisions," European Physics Journal A, Vol. 12, No. 4, 2001, pp. 405-411.

[17] S. K. Charagi and S. K. Gupta, "Coulomb-modified Glauber Model Description of Heavy-Ion Reaction Cross Sections," Physical Review C, Vol. 41, No. 4, 1990, pp. 1610-1618.

[18] S. K. Charagi, "Nucleus-Nucleus Reaction Cross Section at Low Energies: Modified Glauber Model," Physical Review C, Vol. 48, No. 1, 1993, pp. 452-454.

[19] S. K. Patra, R. N. Panda, P. Arumugam and R. K. Gupta, "Nuclear Reaction Cross Sections of Exotic Nuclei in the Glauber Model for Relativistic Mean Field Densities," Physical Review C, Vol. 80, No. 6, 2009, p. 064602.

[20] A. Shukla, B. K. Sharma, R. Chandra, P. Arumugam and S. K. Patra, "Nuclear Reaction Studies of Unstable Nuclei Using Relativistic Mean Field Formalisms in Conjunction with the Glauber Model," Physical Review C, Vol. 76, No. 3, 2007, p. 34601.

[21] B. K. Sharma, et al., "Reaction Cross-Sections for Light Nuclei on ${ }^{12} \mathrm{C}$ using Relativistic Mean Field Formalism," Journal of Physics G: Nuclear Particle Physics, Vol. 32, No. 11, 2006, pp. 2089-2097.

[22] M. D. Estal, M. Centelles, X. Vinyas and S. K. Patra, "Pairing Properties in Relativistic Mean Field Models Obtained from Effective Field Theory," Physical Review C, Vol. 63, No. 4, 2001, p. 044321.

[23] S. K. Patra, M. D. Estal, M. Centelles and X. Vĩnas, "Ground-State Properties and Spins of the Odd $Z=N+1$ Nuclei ${ }^{61}$ Ga- ${ }^{97}$ In," Physical Review C, Vol. 63, No. 2, 2001, p. 024311.

[24] B. A. Ibrahim, Y. Ogawa, Y. Suzuki and I. Tanihata, "Cross Section Calculations in Glauber Model: I. Core Plus One-nucleon Case," Computer Physics Communications, Vol. 151, No. 3, 2003, pp. 369-386.

[25] K. Hagino, N. Rowley and A. T. Kruppa, "A Program for Coupled-channel Calculations with All Order Couplings for Heavy-Ion Fusion Reactions," Computer Physics Communications, Vol. 123, No. 1-3, 1999, pp. 143-152.

[26] L. H. Bradt and B. Peters, "The Heavy Nuclei of the Primary Cosmic Radiation," Physical Review, Vol. 77, No. 1, 1950, pp. 54-70.

[27] S. Barshay, C. B. Dover and J. P. Vary, "Nucleus-nucleus Cross Sections and the Validity of the Factorization Hypothesis at Intermediate and High Energies," Physical Review C, Vol. 11, No. 2, 1975, pp. 360-369.

[28] S. Barshay, C. B. Dover and J. P. Vary, "The Validity of the Factorization Hypothesis for Nucleus-nucleus Cross Sections at High Energies," Physics Letters B, Vol. 51, No. 1, 1974, pp. 5-8.
[29] A. Ingemarsson and M. Lantz, "Geometrical Aspects of Reaction Cross Sections for ${ }^{3} \mathrm{He},{ }^{4} \mathrm{He}$, and ${ }^{12} \mathrm{C}$ Projectiles," Physical Review C, Vol. 67, No. 6, 2003, p. 064605.

[30] A. Ingemarsson and M. Lantz, "Energy Dependence of Proton-Nucleus Reaction Cross Sections," Physical Review $C$, Vol. 72, No. 6, 2005, p. 064615.

[31] C.-T. Liang, Y.-A. Luo, X.-H. Li and C.-H. Cai, "Systematic Analysis of the Reaction Cross Section for $d,{ }^{3} \mathrm{He}$ and ${ }^{4} \mathrm{He}$ as Projectiles," Journal of Physics G: Nuclear Particle Physics, Vol. 36, No. 1, 2009, p. 015102.

[32] H. P. Wellisch and D. Axen, "Total Reaction Cross Section Calculations in Proton-nucleus Scattering," Physical Review C, Vol. 54, No. 3, 1996, pp. 1329-1332.

[33] J. Y. Hostachy, et al., "Elastic and Inelastic Scattering of ${ }^{12} \mathrm{C}$ Ions at Intermediate Energies," Nuclear Physics A, Vol. 490, No. 2, 1988, pp. 441-470.

[34] V. Bernard, et al., "Production of Charged Pions in Intermediate-Energy Heavy-Ion Collisions," Nuclear Physics A, Vol. 423, No. 3, 1984, pp. 511-524.

[35] J. N. De, X. Vinyas, S. K. Patra and M. Centelles, "Nuclei beyond the Drip Line," Physical Review C, Vol. 64, No. 5, 2001, p. 057306.

[36] P. A. Mazzali, et al., "A Neutron-star-Driven X-ray Flash Associated with Supernova SN 2006aj," Nature, Vol. 442, No. 7106, 2006, pp. 1018-1020.

[37] T. A. Thompson, P. Chang and E. Quataert, "Magnetar Spindown, Hyper-energetic Supernovae, and Gamma Ray Burst," Astrophysics Journal, Vol. 611, No. 1, 2004, pp. 380-393.

[38] P. O. Lagage and C. J. Cesarsky, "The Maximum Energy of Cosmic Rays Accelerated by Supernova Shocks," Astronomy and Astrophysics, Vol. 125, No. 2, 1983, pp. 249-257.

[39] J. R. Wilson and R. W. Mayle, "Report on the Progress of Supernova Research by the Livermore Group," Physics Reports, Vol. 227, No. 1-5, 1993, pp. 97-111.

[40] B. S. Meyer, "The r-, s-, and p- Process in Nucleosynthesis," Annual Review of Astronomy and Astrophysics, Vol. 32, 1994, pp. 153-190.

[41] J. M. Pearson, "Nuclear Physics: Energy and Matter," Ch. II, Adam Hilger Ltd, 1986, p. 87.

[42] A. Marinov, et al., "Evidence for the Possible Existence of Long-lived Superheavy Nucleus with Atomic Mass Number A $=292$ and Atomic Number $Z \approx 122$ in Natural Th," International Journal of Modern Physics E, Vol. 19, No. 1, 2010, pp. 131-140.

[43] S. K. Patra, M. Bhuyan, M. S. Mehta and R. K. Gupta, "Superdeformed and Hyperdeformed States in $\mathrm{Z}=122$ Isotopes," Physical Review C, Vol. 80, No. 3, 2009, p. 034312. 